\title{
Genealogías descolonizantes en los feminismos emergentes: el impacto de las mujeres zapatistas.
}

\section{Decolonizing genealogies in the emerging feminisms: impact of zapatistas women.}

Márgara Millán *

A Claudia y Sonia, por ser provocadoras del pensamiento

\section{Resumen:}

En este ensayo pensaré en el momento actual del feminismo, en tanto nombre que damos a las resistencias de mujeres concretas en situaciones específicas, en tensión con el capitalismo global y sus concreciones patriarcales. En ese horizonte, el espacio de lo nacional aparece como un espacio que exige una intencionalidad crítica integral e integradora. Para dar ese paso, los feminismos han tenido que traspasar fronteras identitarias anteriores en un ejercicio de auto-descolonización, proceso en el que ha sido fundamental la emergencia y diálogo con las mujeres indígenas y campesinas. La insurgencia del movimiento zapatista en México y la agenda de discusión que coloca a nivel internacional ha tenido un papel fundamental en la visibilización y enunciación de "las mujeres que luchan", lugar y palabra que en los años recientes se han ido configurando como una plataforma de lucha antisistémica (anti-capitalista y antipatriarcal), descolonizando ilusiones de la modernidad.

Palabras clave: feminismos, descolonización, modernidad, América Latina, mujeres zapatistas

\section{Abstract}

\footnotetext{
*Socióloga y Doctora en Antropología Social, profesora titular en la Facultad de Ciencias Políticas y Sociales de la Universidad Nacional Autónoma de México. Ha dirigido lo sproyectos de Modernidades alternativas y nuevo sentido común: anclajes de una modernidad no capitalista (2011-2014), y Modernidades alternativas y prefiguraciones de lo político (2015 a 2018). Es parte de la Red de Feminismos Descoloniales.
} 
At Feminism at the present moment, as a name that we give to the resistances of specific women in specific situations, demands an integral and integrating critical intentionality on the Nation and the national space. In order to take that step, feminisms have had to cross identity boundaries in an exercise of self-decolonization. The zapatista indigenous uprising in México and the following political agenda has been essencial for new visibility and a new politics of enuntiation of "women in struggle", placement and speech space that in the recent years have been formulating an antisistemic platform, anti-capitalist and antipatriacal, decolonizing modernity illusions.

Key words: feminisms, decolonization, modernity, LatinAmerica, zapatista women

Fecha de recepción: Marzo 2019

Fecha de aprobación: Junio 2019

\section{La dialéctica nacional / global}

¿Es la pregunta por lo nacional pertinente al estudiar el feminismo? ¿Hay una marca del lugar desde donde se articula y emite el discurso feminista? Sin duda hoy es claro que los feminismos locales aportan a una teoría feminista situada y compleja, que prefigura una lucha antisistémica global. Ello porque esa es la forma en la que hoy los movimientos de mujeres en todo el mundo reconocen la vinculación existente entre las violencias locales que enfrentan y el sistema a la vez capitalista y patriarcal.

Así, al mismo tiempo que el feminismo actual se nutre de luchas situadas y locales, de historicidades concretas, la condición de globalización de los movimientos y de las ideas, de las figuras e imágenes de los movimientos sociales, han hecho que el feminismo contemporáneo suene, resuene y hable en varias lenguas. Que las improntas de lo que dice la calle, a través de la creación de consignas como iparamos para cambiarlo todo! den la vuelta al mundo e intensifiquen lo que podríamos denominar un dialógico espíritu de época.

En este ensayo pensaré que en el momento actual, el feminismo en tanto nombre que damos a las resistencias de mujeres concretas en situaciones específicas, exige una intencionalidad crítica integral e integradora. Para dar ese paso, los feminismos han tenido que traspasar fronteras identitarias anteriores en un ejercicio de auto-descolonización, han tenido que "desclasarse", integrarse, mezclarse. Me parece que estamos en un momento particular y distinto de la lucha feminista, un momento en el cuál, de la solidaridad entre luchas específicas, se transita a la enunciación de una lucha global antisistémica.

En México, esta tendencia general, que denominaré la descolonización de los feminismos, ha tenido un impulso masivo a partir del levantamiento insurgente del 
zapatismo indígena en el sur del país. Ubicaré el momento histórico donde esto sucede como el del inicio de la caída del neoliberalismo y del relanzamiento de la crítica social si se quiere de una izquierda - que habla desde la clase al mismo tiempo que desde la etnia silenciada y acotada, lo mismo desde la subalternidad social que desde la otredad cultural y civilizacional. Y trataré de mostrar cómo ese discurso de y sobre las mujeres indígenas, que inicia en 1994 desde la selva lacandona, se entrelaza en los últimos años con los movimientos amplios de mujeres en las principales ciudades del país, y con la realidad de lucha en contra del feminicidio y de las desapariciones.

Lo particular del momento presente en los feminismos que toman las calles y redactan manifiestos es la confluencia de agendas, de heterogeneidades de clase, raza, edad, diversidad sexual. No sin tensiones y conflictos, parecería configurarse un sujeto político emergente múltiple y complejo que responde también a la emergencia que hoy vivimos: la guerra contra las mujeres, que se manifiesta en el feminicidio, la guerra contra las comunidades que destruye sus territorios y formas de vida, la guerra contra los y las jóvenes, la guerra contra el planeta. Ello apunta hacia una reacción múltiple que descubre en la organización capitalista, patriarcal y colonizada de lo social-cultural el principal enemigo. Esta confluencia de espacios y temporalidades hace que lo local retumbe en lo global cada vez más, es decir, que lo local se globalice y se reconozca en estas situaciones locales de violencia, despojo, injusticia, una situación compartida globalmente.

Ahora bien, la dimensión local contribuye a dibujar y comprender la manera en la que la colonización - de la historia, del imaginario, del género - transcurre entreverado con las culturas nacionales, con el mito de origen que cada nación ha debido "inventar" para afirmarse como Nación. En América Latina -como en otras partes del mundo colonizado- el estado-nación ha debido construirse como una nación de estado ${ }^{1}$, a manera de construir identidades más o menos homogéneas y participar del ordenamiento global capitalista que va a ir funcionando en el mercado.

La cultura nacional es así un horizonte a deconstruir desde una perspectiva crítica y descolonizadora feminista. El lugar de las mujeres es también un lugar o una serie de (o)posiciones asignadas por la nación-estado. Cuando el feminismo habla de interseccionalidad y de descolonización se refiere a los procesos de racialización o etnización y de clase social dentro de un continente específico, que es la nación, la cultura nacional, los nacionalismos.

La pregunta entonces por "lo nacional" adquiere relevancia porque apunta a cómo los feminismos se posicionan frente y dentro de procesos específicos de hegemonía política y cultural, develando las relaciones internas de dominación / colonialidad en las cuáles intervienen, focalizando la colonización interna, concepto que rescatamos de la teoría latinoamericana de los años 70 s. $^{2}$

\footnotetext{
${ }^{1}$ Noción que retomo de la obra del filósofo ecuatoriano-mexicano Bolívar Echeverría, ver "Cuestionario sobre lo político", conversación con Luis Corral y José Ron (1980), en Bolívar Echeverría, ensayos políticos, editado por Ministerio de Coordinación de la Política y Gobiernos Autónomos Descentralizados, Quito, 2011.

${ }^{2}$ Pablo González Casanova en La democracia en México, México, Editorial Era, editado por primera vez en 1965, enfrenta la cuestión de una sociedad plural, multiétnica, reconocida
} 
La nación es así un espacio formador de una identidad, al mismo tiempo que de una narrativa cultural hegemónica que asigna los lugares que ocupamos entre mujeres, parafraseando el libro de Rita Segato, podríamos pensar en La Nación y sus otras ${ }^{3}$.

La hegemonía cultural nacional opera como matriz incluso para los discursos críticos ilustrados, entre ellos el feminismo. Para comprender cómo opera esa matriz nos hacen falta categorías otras, ya que las caracterizaciones políticas más generales (feminismo liberal, socialista, neoliberal) estarían describiendo ciertos lugares de enunciación, pero oscureciendo otros. Lo mismo estaría sucediendo con categorizaciones como las de feminismo de la diferencia, de la igualdad, autónomos, institucionales, que sin dejar de ser descriptiva y políticamente útiles, no alcanzan a decir la crítica pluriversal presente en el momento actual del movimiento feminista. Se trata de feminismos que políticamente se pueden diferenciar pero que quizá comparten las certezas "nacionalistas", las identidades fuertes de la construcción nacional, y con ello, la asunción de una cierta noción de historicidad y de temporalidad, que deja fuera la coetaneidad de mundos no capitalistas, como lo plantea Rivera Cusicanqui ${ }^{4}$, sin poder reconocer en estas otras temporalidades una coetaneidad activa e interpelante de la modernidad capitalista.

La emergencia desde hace al menos tres décadas de los feminismos adjetivados son un síntoma de esta intencionalidad crítica descolonizante: el feminismo chicano, afroamericano, y desde ahí, según el contexto de la emergencia, el feminismo islámico, el feminismo indígena, los feminismos callejeros, son formas de dar cuenta no sólo de la pluralidad del sujeto del feminismo, sino de cómo la narrativa nacional construye una matriz hegemónica de sentido que ordena el lugar de las otras dentro de la Nación.

En este sentido, las emergencias localizadas de feminismos adjetivados son procesos fragmentarios de descolonización del Feminismo en tanto discurso unitario, ilustrado y modernizante, ya que problematizan la univocidad de su capacidad

también por René Zavaleta Mercado en el concepto de lo multisocietal, pero es la relectura de Silvia Rivera Cusicanqui la que en realidad propone una lectura del colonialismo interno que se dirige más claramente a un diagnóstico del colonialismo interno y a un horizonte descolonizador. Para ella es fundamental la estratificación y las mediaciones que van formando la "cadena de relaciones de dominio colonial", las "exclusiones eslabonadas" que componen el mundo mestizo. Como afirma Sinclair Thomas: "El mundo del mestizaje -marcado por una inescapable ambivalencia- se vuelve un espacio crítico en el cuál uno (a) es tanto victimizado(a) como víctima. En este punto encontramos otra de las perspectivas más fuertes y profundas en la obra de Silvia. Su sensibilidad hacia la violencia cotidiana en los sectores medios permite una visión más cruda del mestizaje que contrasta con la idealización nacionalista que ve en él un espacio de ascenso social y de armonía entre clases y grupos étnicos[...]Sinclair Thomas, en el prólogo a Silvia Rivera Cusicanqui, Violencias (re) encubiertas en Bolivia, 2010, La Paz, Ed. La mirada salvaje, p. 14-16.

El mestizaje es un dispositivo que se va re-inventando en distintos conceptos, como por ejemplo el de ciudadanización, en el neoliberalismo. El "ideologema del mestizaje" oculta esas relaciones de colonialismo interno, y ello debe de ser un campo de deconstrucción para el feminismo crítico. ${ }^{3}$ Me refiero al libro La Nación y sus Otros. Raza, etnicidad y diversidad religiosa en tiempos de Políticas de la Identidad, 2007, Buenos Aires, Ed. Prometeo.

${ }^{4}$ Ver Silvia Rivera Cusicanqui, Un mundo ch'ixi es posible. Ensayos desde un presente en crisis, 2018, Buenos Aires, Ed. Tinta Limón. También en Ch'ixinakaxUtxiwa. Una reflexión sobre prácticas y discursos descolonizadores. 2010, Buenos Aires, Tinta Limón 
heurística y epistémica, abriendo espacios para el análisis de la politicidad concreta de las mujeres culturalmente situadas.

De manera análoga a cómo se ha construido la noción del "tercer mundo" o de las mujeres de las periferias por el "feminismo occidental" Sur global, para hablar de la dialéctica de producción de sentido y ordenamiento de las subalternidades que ocurre en el espacio de lo global, podemos estudiar la construcción de "la nación y sus otras", siendo este el espacio preciso de producción y actualización del colonialismo interno, del cual el feminismo no resulta ajeno.

En México, esta dialéctica del colonialismo interno al interior del feminismo a través de las narrativas nacionales quedó expuesta de múltiples formas con el levantamiento indígena zapatista, abriendo un horizonte nuevo a las resistencias feministas, cuyos principales frentes crítico enumero a continuación.

\section{El indigenismo como mirada (colonial):}

El estado mexicano construyó a partir de la revolución de inicios del siglo XX una idea salvífica dirigida a los indígenas, que operó a través de la imposición de una sola temporalidad, la del estado nación, al tiempo que un reconocimiento histórico-cultural del pasado (anacrónico) de lo pre-hispánico.

Así, el reconocimiento y recuperación memorística y museográfica de los antecesores "pueblos originarios" dio un lugar en la identidad nacional al "pasado indígena", incluso permitió la admiración y nostalgia por los modos de vida aún conservados en las comunidades indígenas. Pero al mismo tiempo, y por el mecanismo de la distancia e imposibilidad histórica de su actualización, se empeñó en desconocer las formas coetáneas de organización, producción y soberanía de esas comunidades indígenas, no solo como formas propias de existencia, sino como formas interpelantes y posibles en la propia modernidad nacional. El efecto de esto fue la invisibilización de las formas culturales y políticas indígenas, y la visibilización del indígena como "pobre". Los indígenas perdieron pertenencia comunitaria, idioma, identidad, a los ojos del Estado y de la cultura hegemónica mestiza, que en su afán progresista y su certeza de univocidad de futuro, establecieron políticas asimilacionistas para "mexicanizar" y ciudadanizar al indio, es decir, des-indianizarlo.

Dentro de esta perspectiva, las mujeres indígenas quedaron subsumidas en una caracterización de "la ignorancia, la superstición, lo ladino". En su conjunto, lo indígena fue -y sigue siendo - feminizado e infantilizado. Es la población atrasada del país, por ello incluso se habla de la diferencia entre el norte emprendedor y el sur atrasado de México; el estado lanza políticas para su desarrollo e integración, políticas que operan a través de su conversión a lo mestizo / criollo, su proceso de blanqueamiento.

Las mujeres "indígenas" son desvalorizadas por "monolingües", al tiempo que enaltecidas por "preservar la cultura originaria", pero nunca consideradas como

\footnotetext{
${ }^{5}$ Ver Chandra Mohanty, "Bajo los ojos de Occidente: academia feminista y discursos coloniales", en Liliana Suárez y Rosalva Aída Hernández, eds. Descolonizando el feminismo. Teorías y prácticas desde los márgenes, 2008, Madrid, Ed. Cátedra, pags. 117-163
} 
interlocutoras políticas cabales. Y en el cruce de diversas ideologías, tanto feminismo como acción estatal recomiendan la familia pequeña, y en algunos momentos, imponen políticas de contención de la natalidad.

Este proceso o dispositivo ha sido denominado por Silvia Rivera Cusicanqui, para la realidad boliviana, como la "miserabilización del indio", sujeto que queda sin cultura, con la sola pertenencia a la "pobreza extrema", población "vulnerable", sujeta a las políticas de los programas sociales estatales. Proceso que ocurre simultáneamente al ideologema del mestizaje, como el mito de la superación de lo indio en la matriz nacional criolla (Rivera Cusicanqui, 2010)

Para el indigenismo como política estatal hacia los indígenas, se trata de tutelar a sujetos que no son considerados plenos políticamente, es decir, sujetos que no tienen agencia propia ni capacidad de auto-organización; sujetos siempre instrumentalizados por "otro" cuando se le oponen al estado.

Esta narrativa usa la alocución de "nuestros indígenas" como si fueran una reserva a la cual hay que proteger y cuidar, pero nunca, como indígenas que son, una parte activa, propositiva, creativa de lo nacional. Lo nacional aparece así como un factum, algo dado y cerrado, constituido en un momento preciso de la historia como ordenador del todo de la nación, que para integrar debe modernizar.

$\mathrm{He}$ analizado en otro lugar ${ }^{6}$ el impacto que el alzamiento insurgente indígena tuvo en los feminismos en México a través del análisis de las reacciones y posicionamientos de tres revistas feministas mexicanas: Fem, La Correa Feminista y Debate Feminista. Encontramos la fuerza del indigenismo en las tres publicaciones, ya sea bajo el dispositivo de la esencialización y romantización de la mujer o por el contrario, a través del dispositivo de la miserabilización, sobre todo en cuanto a su agencia propia (Millán, 2009)

Así, el enigma de la presencia mayoritaria de las mujeres en la rebelión, su visibilización en todos los frentes del movimiento: insurgentas, comandantas, bases de apoyo, no propiciaba la escucha atenta y lo que podríamos denominar el diálogo intercultural entre feminismos distantes.

\section{Lo laico como frontera (de clase):}

La herencia liberal republicana más fuerte en la construcción de la Nación Mexicana ha sido la lucha por la laicidad del estado, y por la secularidad de la vida pública. Distinto a muchos estados del continente, tanto del sur como del norte de las Américas, México se decantó por el lado secular y laico de la vida pública.

La herencia Juarista ${ }^{7}$ se pone en duda a partir del triunfo del partido de derecha (Partido de Acción Nacional, PAN) en el año 2000. Ese año es de inflexión en términos de las narrativas de la nación: es el año en que los Zapatistas llegan al Congreso,

\footnotetext{
${ }^{7}$ Benito Juárez es el Presidente de México que lucha contra el Imperio Francés e instaura la República en la segunda mitad del siglo XIX. Representante del pensamiento liberal, se le reconoce también el haber quitado el poder que la Iglesia mantenía sobre el estado y la política.
} 
hablan a través de la voz de la Comandanta Esther y proponen un programa de refundación nacional. Esther inicia su alocución diciendo: soy Mexicana, soy mujer y soy indígena. La distancia insalvable entre sus palabras y la capacidad de comprensión que de ellas tuvo la clase política Mexicana fue abismal, en el sentido de Boaventura de Sousa Santos ${ }^{8}$. No hubo posibilidad de traducción. El estado mexicano no inicia un proceso de refundación de la nación. Lo que ocurre es que se da inicio a una transición política unipartidista (setenta años de dictadura perfecta del Partido revolucionario Institucional, PRI) que deja intocada la relación con los pueblos originarios y sus demandas.

El movimiento indígena zapatista cultiva cuatro tradiciones de lucha y pensamiento: la lucha agraria, de larga duración entre el campesinado del sureste mexicano, con logros importantes en la reforma agraria posrevolucionaria. Esa es la herencia de Zapata. La lucha de liberación nacional, heredera de la revolución cubana, del sueño latinoamericanista, es también la de la guerrilla, en su caso, de línea maoísta, y que en esa región anida abriéndose a un proceso de aculturación, dando como resultado la paradoja de un ejército que da paso a la organización civil, y mandata la paridad de género. La tercera tradición es la teología india, la palabra de dios. Inculcada por la diócesis de San Cristóbal de Las Casas, Chiapas, da paso también a un proceso de inculturación: la teología de la liberación encarna en la teología india, donde las comunidades diversas traducen la Biblia a su propia lengua, literal y metafóricamente. Desde entonces, parte importante del movimiento va a ser esa vivencia de la liberación a través del sacrificio, esa actualización en el presente de la gesta descrita en las sagradas escrituras. Y por último, el camino de la tradición, es decir, de la actualización constante que el mundo indígena hace de su propia tradición, de su entendimiento del mundo y de su lugar en él, de su ontología y cosmovisión: la relación con el entorno, con la vida presente y pasada, con las cuatro esquinas del universo; de esa tradición se actualiza la responsabilidad y obligación que se tiene con el todo, la idea de ser parte, la idea de lo común.

Parte del feminismo ilustrado mexicano entra en contradicción profunda con el sentido de agencia que se desprende de la pertenencia y afirmación comunitaria de las mujeres indígenas en lo general, y zapatistas en lo particular.

La agencia de las mujeres indígenas apareció a los ojos de algunas corrientes feministas como una agencia dependiente e instrumentalizada por la dirigencia del movimiento, o incluso por la Iglesia ${ }^{9}$, influencias externas y patriarcales que de alguna

${ }^{8}$ Ver por ejemplo Para descolonizar Occidente. Más allá del pensamiento abismal.

${ }^{9}$ Es así como enmarco la discusión que se dio entre la renombrada antropóloga y activista feminista Marta Lamas y el Subcomandante Marcos en relación a la reforma del código penal de Chiapas que volvía a penalizar el aborto. La antropóloga sugería un tutelaje del obispo de San Cristóbal de las Casas, Chiapas, don Samuel Ruíz sobre el movimiento insurgente y en lo particular sobre las mujeres, en sentido de la penalización, a lo que el Subcomandante sostiene una contundente respuesta poniendo en duda que lo prioritario para la agenda de las mujeres indígenas sea la despenalización del aborto, ya que lo que solicitan son clínicas para que sus hijos/as sobrevivan, argumentando también que cuando las mujeres indígenas querían interrumpir su embarazo, sabían cómo hacerlo (Millán, 2014) 
manera "limitaban" el feminismo de las mujeres indígenas. La interpelación cultural que el movimiento en su conjunto y en particular las mujeres indígenas zapatistas establecían al mundo moderno de la emancipación feminista no podía ser reconocida, y en su lugar aparecían representaciones colonialistas de las mujeres zapatistas en lucha. No se deja desestabilizar o descentrar por la interpelación comunitaria que el zapatismo muestra. La interpretación que prevalece sigue considerando a las mujeres indígenas como no agentes, instrumentos de la voz masculina de los voceros protagónicos del movimiento; a tal punto, que se le escapan las palabras y las inflexiones de las mujeres indígenas, su propia forma de enunciar las desigualdades que habitan, la complejidad de la interacción con y entre mujeres divididas por clase, cultura, cosmovivencia.

Indigenismo y laicismo en la cultura mexicana son vectores del poder de la nación pero también -y por ello mismo- de la modernidad capitalista y su ciudadanía liberal. Son ejes significantes ambiguos, ya que reconocen derechos, pero al mismo tiempo homogenizan a los sujetos y eluden las diferencialidades. Los feminismos críticos tienen que hacerse cargo de esos legados, y tejer puentes (articular luchas) entre distintas posicionalidades, lo cual sólo puede realizarse a través de un proceso de descolonización constante. Es decir, los posicionamientos anticapitalistas y descolonizantes deben ser capaces de atravesar los contenidos feministas de forma histórica y contextualizada, y ello implica una deconstrucción crítica de lo "nacional" y de la ciudadanía liberal.

A partir de estas tensiones, pero también de estos acercamientos, se va generando en México un feminismo "abajo y a la izquierda", que se disemina de forma interclasista, intergeneracional, interétnica. Este cruce de fronteras es auspiciado por un conjunto de encuentros organizados desde el EZLN, que durante estos 25 años de irradiación descolonizadora se deja ver en el feminismo mexicano ${ }^{10}$, y sobre todo en las jóvenes. Se trata de un feminismo heterogéneo que va conjuntando las demandas en contra de las violencias hacia las mujeres, en contra de las prácticas patriarcales, al tiempo que anticapitalista, sobre todo en el sentido de la lucha contra el extractivismo y el despojo de los territorios. Poco a poco, el crisol de luchas que componen el terreno de las resistencias anticapitalistas se va viendo atravesado por esta intencionalidad feminista.

Así, el terreno de articulación de luchas y de visibilización de opresiones comunes, han ido desarrollando, más allá de la solidaridad, la noción de una lucha compartida, contra el capitalismo y el patriarcado, es decir, entre movimientos sociales y feminismos.

\footnotetext{
${ }^{10}$ El EZLN desde muy pronto inició una convocatoria a nivel nacional e internacional en su territorio que fue provocando una serie de encuentros, construyendo una comunidad internacional, y convirtiéndose en un centro cosmopolita de pensamiento crítico y de relanzamiento de un discurso que desde el inicio incorporó a las mujeres y su condición. Desde la Convención Nacional convocada en 1996 en Guadalupe Tepeyac, corazón del territorio autónomo, pasando por el Encuentro Intergaláctico contra el neoliberalismo y por la humanidad, y posteriormente por los encuentros y seminarios así como los CompArte y ConCiencias, y últimamente, el encuentro de cine y el encuentro internacional de mujeres, ambos en 2018.
} 
¿Es este un fenómeno global, el de la descolonización del feminismo ilustrado, su articulación con las luchas anticapitalistas de todo tipo: antiextractivismo, defensa territorial, contra la desaparición y la violencia, por ejemplo?

Para Maristella Svampa, que ha pensado con consistencia el modelo contemporáneo del extractivismo en Latinoamérica y que califica al momento actual como de "exacerbación del metabolismo social del capitalismo", es decir, aumento de su capacidad destructiva del entorno, ya que todo se convierte en recursos productivos, asistimos hoy a una convergencia de los feminismos ecológicos y ambientalistas con los feminismos populares, y ello ocurre en el terreno de las luchas sociales (Svampa, 2018)

Para Cinzia Arruzza, Tithi Bhattacharya y Nancy Fraser (2019), autoras del Manifiesto de un feminismo para el $99 \%$ en contra del feminismo corporativo, que se encuentra muy visible en el norte del continente americano, y cuya agenda es la de "empoderémonos", incluso a través de las políticas del micro-crédito, más allá del horizonte de éxito del neoliberalismo y frente a él, tendríamos que re-lanzar el ethos radical y transformador del feminismo. Reimaginar la justicia de género de forma anticapitalista parece ser la ruta planteada por los recientes movimientos de mujeres del 2016 hasta ahora. Este Manifiesto nos llama a pensarnos como movimiento feminista internacionalista, antiracista, ecologista, anticapitalista y anti-imperialista, que ha resignificado el 8 de marzo, y que ha relanzado una noción de militancia, incluso poniendo a actuar instrumentos de clase como el paro y la huelga. Releva de forma muy clara algo que ha estado presente en las luchas y las reflexiones del feminismo, que lo lleva a implicarse con el todo, a diseminar su intencionalidad crítica y transformativa al todo social, y desde un sujeto colectivo diverso, el sujeto del feminismo contemporáneo (Millán, 2014).

Implicación con el mundo que hace "temblar la tierra", metáfora del zapatismo pero también del movimiento en el sur Latinoamericano ${ }^{11}$ que nos hace pensar en el salto del propio sujeto que está convocando a la acción, al "incendio" como acción, desde las mujeres.

\section{Nuevos horizontes, nuevas textualidades}

Me parece que este manifiesto, junto con una serie de textos elaborados para autoconvocarnos a las movilizaciones cada vez más globales de \#Ni una más; \#Vivas nos queremos; \#Ni una menos, \#Nos tocan a una, nos tocan a todas, por mencionar las frases que han reconfigurado las luchas, nos ofrecen un material invaluable para comprender el "espíritu" de la politicidad actual del feminismo local/global, sus nuevas tendencias, orientaciones y textualidades.

Este conjunto de luchas que se han ido articulando es lo más cercano a la interseccionalidad entendida como praxis. Se compone de múltiples elementos críticos que se afianzan en las diferentes realidades constitutivas del sujeto social "mujeres" de frente al sistema que hoy domina. Resulta así que, parafraseando a Rita Segato:

11Ver Verónica Gago, "La tierra Tiembla", Critical Times, Vol 1, N. 1, 2018, Berkeley, Internacional Consortium of Critical Theory. Pp. 178-184 
"refundar el feminismo para refundar la política" va teniendo distintas textualidades, un vocabulario, y las prácticas de los encuentros, así como tomar las calles, las plazas, ir al paro y a la huelga. Todo ello desde un retejer la comunidad con un cosmos propio y contrario al proyecto histórico del capital ${ }^{12}$.

Formando parte de ese acervo, en una alianza que no siempre se explicita pero que sugiero, se encuentra el texto que a continuación transcribo íntegramente, el de la clausura del Primer Encuentro Internacional Político, Deportivo y Cultural de mujeres que luchan, convocado por las mujeres zapatistas en marzo del 2018 en el Caracol Morelia, en el municipio de Altamirano, Chiapas, territorio zapatista, y al cuál acudieron cerca de ocho mil mujeres de todo el mundo.

Lo reproduzco de forma íntegra porque merece una difusión y una lectura insistente. Es a través de este tipo de textos que podemos acercarnos a la subjetividad de lucha de la diversidad de la que hablamos.

\section{Palabras de las mujeres zapatistas en la clausura del Primer Encuentro Internacional, Político,Artístico, Deportivo y Cultural de Mujeres que Luchan en el Caracol Zapatista de la Zona Tzotzchoj}

Buenas noches, buenos días, buenas tardes, compañeras y hermanas que luchan, donde quiera que se encuentren.

Hermanas y compañeras que nos acompañaron en este primer encuentro internacional de mujeres que luchan.

Vamos a decir unas pequeñas palabras a nombre de todas nosotras, las mujeres zapatistas de los cinco caracoles.

Queremos agradecer a las compañeras ciudadanas de los equipos de apoyo que lo sabemos que bien que se chingaron en los correos, el registro, la organización del transporte y el acomodo de los horarios y lugares de las actividades.

Queremos también saludar aquí a nuestras compañeras zapatistas que no pudieron venir aquí en este encuentro y quedaron pendientes para que nosotras pudiéramos llegar.

Mismo a nuestros compañeros que tuvieron que quedar para cuidar nuestra familia, nuestros animales, nuestras casas, nuestros cuarteles, nuestros campos, y que estuvieron pendientes por si los malos gobiernos hacen alguna maldad contra del encuentro.

Pero nuestras palabras finales son especiales para ustedes, hermanas y compañeras mujeres que luchan.

Agradecemos con todo nuestro corazón humilde y sencillo, con resistencia y rebeldía, la participación de todas ustedes, mujeres que luchan en los cinco continentes del mundo.

Tanto las que están aquí, como las que están pendientes de lo que aquí pasó.

\footnotetext{
12"Retejer comunidad significa alistarse en un proyecto histórico que se dirige a metas divergentes con relación al proyecto histórico del capital. Aquí la religión o lo que he llamado "cosmos propio" juega un papel considerable", Rita Segato, Manifiesto en cuatro temas, Critical Times, Vol 1, N. 1, 2018, Berkeley, Internacional Consortium of Critical Theory. Pags 221 y 222
} 
Agradecemos sus oídos, sus miradas, sus palabras, sus talleres, sus pláticas, su arte, sus videos, sus músicas, sus poesías, sus cuentos, sus teatros, sus danzas y bailables, sus pinturas, sus cosas raras que ni sabíamos qué son, y todo lo que nos trajeron para que nosotras conocemos y aprendemos de sus luchas.

Lo tomamos todo como un regalo muy valioso que vamos a cuidar y vamos a hacer más grande, porque lo vamos a llevar a nuestras comunidades y pueblos, para que más mujeres zapatistas compartan con nosotras su regalo que nos dieron.

Lo recibimos con respeto y cariño porque todas ustedes hicieron un gran esfuerzo para viajar desde sus lugares de lucha, desde sus tiempos y modos, desde sus mundos, y llegar a este encuentro que no sabemos todavía si salió bien o salió mal.

Ya hemos visto de algunas de las cosas que entregaron en la mesa de críticas a nosotras. Falta que leemos todo y falta que entre todas nosotras las analizamos. En esa caja encontramos una carta que dice algo que creemos que nos viene tocando a todas. Una compañera les va a leer.

(se lee una carta de las familiares de los ausentes de Ayotzinapa, pidiendo que no se les deje solos, porque el mal gobierno quiere cerrar el caso y dejarlo en el olvido).

No las hemos revisado todas las notas, pero les aseguramos nuestro compromiso a corregir lo que nos señalan que está mal, y a mejorar lo que nos dicen que no está cabal.

Sí decimos claro que, hasta ahora, la gran mayoría son críticas por errores y fallas que tenemos en la organización.

Les decimos que vamos a tomar en cuenta todas sus críticas para mejorar la próxima vez, si es que hay una próxima vez. Todas esas críticas, así como nuestras palabras que trajimos en estos días, lo vamos a publicar en la página de enlace zapatista para que ahí todas ustedes pueden ver.

Pero como quiera, así en general, queremos saber lo que piensan todas.

Entonces les preguntamos, compañeras y hermanas:

¿Será que salió un poco bien?

¿O será que salió mal?

Bueno, aquí apuntamos que aunque respondan que salió un poco bien o que salió mal, pues les vamos a contar, sinceramente, una cosa que les pedimos que queda aquí entre nosotras, como mujeres que somos y que luchamos. O sea que no lo vayan a andar contando, sobre todo con los hombres.

Pues la verdad, hermanas y compañeras, es que sufrimos mucho porque no sabemos cómo vamos a hacer.

Es la primera vez que como mujeres que somos, solas, organizamos un encuentro así.

Y lo organizamos desde abajo, o sea que primero hicimos reuniones y discusión en nuestros colectivos en los pueblos y comunidades. Luego en las regiones, luego en las zonas y luego ya de las 5 zonas juntas.

$Y$ pues ya ven ustedes que entre mujeres pues tardamos para hacer un acuerdo pequeño, pues peor si es un acuerdo así de grande como el de hacer este encuentro.

Tardamos meses y así hasta que llegó el acuerdo de todas, porque es que, sí vamos a hacer, es porque tenemos que hacer entre todas, en colectivo. 
$Y$ pues no hay libro o manual para hacer esto.

Y ni modo de preguntarle a los compañeros porque ellos tampoco saben cómo hacer, porque, como ya dijimos, nunca se ha hecho algo así antes.

Entonces pues entre nosotras mismos tenemos que buscarle cómo hacer.

Entonces pues estamos con el pendiente todo el pinche día y toda la pinche noche. Acaso dan ganas de comer, no. Y no entra el sueño.

$Y$ estamos preocupadas por si va a salir bien o va a salir mal.

Nos preocupamos como zapatistas de por sí, pero también como mujeres.

Porque nosotras las invitamos. Entonces va en nuestra cuenta si sale mal o bien.

Que dónde duermen, que dónde comen, que dónde se bañan, que dónde van al baño, que el sonido, que la luz, que el agua, que si se enferman, que qué les vamos a decir, que cómo les hablamos, que cómo las escuchamos y miramos.

Entonces, de corazón les pedimos disculpas por los errores y fallas que tuvimos en esto. Seguro la próxima vez, si es que hay, ya no nos sale tan mal como nos critican.

Porque pensamos que lo más importante es, primero, que estén un poco bien aquí y que se sientan a gusto.

Pero también es importante que miramos y escuchamos a todas, porque si no de balde hicieron la chinga de venir hasta acá y pues lo justo es que escuchemos y miremos a todas. Aunque estemos o no estemos de acuerdo con lo que dicen.

Entonces pues no basta un colectivo para organizar todo eso. Por eso llegamos aquí más de 2 mil mujeres zapatistas de los cinco caracoles.

Y tal vez no bastó, porque ustedes son como cinco mil, aunque algunas dicen que 8 mil y otras dicen que 9 mil.

A saber cuántas mujeres que luchan llegamos en estos días, pero creemos que podemos estar de acuerdo en que somos un chingo.

Y no pensamos si van a llegar tantas porque acá está retirado y no hay comodidades.

Viera que sabemos que son tanto así, pues tal vez llegamos más mujeres zapatistas y así podríamos abrazarlas a todas y cada una y poder decirles en personal lo que ahora les decimos en colectivo.

Vendríamos seis mujeres zapatistas para cada una de ustedes: una pichita (que así les decimos a las que acaban de nacer), una niña, una jóvena, una adulta, una anciana y una finada.

Todas mujeres, todas indígenas, todas pobres, todas zapatistas que te abracen fuerte, porque es el único regalo que podemos darte de vuelta.

Pero como quiera has de cuenta, hermana y compañera, que esto que estamos diciendo aquí, te lo está diciendo una mujer zapatista mientras te da un abrazo y te dice al oído, en tu lengua, en tu modo, en tu tiempo:

"no te rindas, no te vendas, no claudiques"

Que así, con estas palabras es que te decimos

"gracias hermana. Gracias compañera". 
Hermanas y compañeras:

Este día 8 de marzo, al final de nuestra participación, encendimos una pequeña luz cada una de nosotras.

La encendimos con una vela para que tarda, porque con cerillo rápido se acaba y con encendedor pues qué tal que se descompone.

Esa pequeña luz es para ti.

Llévala, hermana y compañera.

Cuando te sientas sola.

Cuando tengas miedo.

Cuando sientas que es muy dura la lucha, o sea la vida,

Préndela de nuevo en tu corazón, en tu pensamiento, en tus tripas.

$Y$ no la quedes, compañera y hermana.

Llévala a las desaparecidas.

Llévala a las asesinadas.

Llévala a las presas.

Llévala a las violadas.

Llévala a las golpeadas.

Llévala a las acosadas.

Llévala a las violentadas de todas las formas.

Llévala a las migrantes.

Llévala a las explotadas.

Llévala a las muertas.

ella.

Llévala y dile a todas y cada una de ellas que no está sola, que vas a luchar por

Que vas a luchar por la verdad y la justicia que merece su dolor.

Que vas a luchar porque el dolor que carga no se vuelva a repetir en otra mujer en cualquier mundo.

Llévala y conviértela en rabia, en coraje, en decisión.

Llévala y júntala con otras luces.

Llévala y, tal vez, luego llegue en tu pensamiento que no habrá ni verdad, ni justicia, ni libertad en el sistema capitalista patriarcal.

Entonces tal vez nos vamos a volver a ver para prenderle fuego al sistema.

$Y$ tal vez vas a estar junto a nosotras cuidando que nadie apague ese fuego hasta que no queden más que cenizas.

Y entonces, hermana y compañera, ese día que será noche, tal vez podremos decir contigo:

"bueno, pues ahora sí vamos a empezar a construir el mundo que merecemos y necesitamos".

Y entonces sí, tal vez, entenderemos que empieza la verdadera chinga y que ahorita como quien dice que estamos practicando, entrenando pues, para ya estar sabedoras de lo más importante que se necesita.

$Y$ eso que se necesita es que nunca más ninguna mujer, del mundo que sea, del color que sea, del tamaño que sea, de la edad que sea, de la lengua que sea, de la cultura que sea, tenga miedo. 
Porque acá sabemos bien que cuando se dice "iya basta!" Es que apenas empieza el camino y que siempre falta lo que falta.

Hermanas y compañeras:

Aquí, delante de todas las que somos aquí y las que no están pero están con el corazón y el pensamiento, les proponemos que acordemos seguir vivas y seguir luchando, cada quien según su modo, su tiempo y su mundo.

¿Están de acuerdo?

Bueno, cuando hacemos este escrito no lo sabemos si responden sí o no, pero paso a la siguiente propuesta:

Como ya lo vimos y escuchamos que no todas están contra el sistema capitalista patriarcal, pues respetamos eso y entonces proponemos que lo estudiemos y lo discutamos en nuestros colectivos si es que es cierto que el sistema que nos imponen es el responsable de nuestros dolores.

Si es que sale que sí es cierto, pues entonces, hermanas y compañeras, saldrá otro día el acuerdo de que luchamos contra el patriarcado capitalista y contra cualquier patriarcado.

Y claro decimos que contra cualquier patriarcado, no importa qué idea tenga, no importa cuál sea su color o su bandera. Porque nosotras pensamos que no hay patriarcado bueno y patriarcado malo, sino que son lo mismo contra nosotras como mujeres que somos.

Si sale que no es cierto, bueno, como quiera nos vamos a estar viendo para luchar por la vida de todas las mujeres y por su libertad y que ya cada quien, según su pensamiento y lo que mira, pues va construyendo su mundo como vea mejor.

¿Están de acuerdo de, en sus mundos y según sus modos y tiempos, estudiar, analizar, discutir y, si se puede, acordar nombrar quién o quiénes son los responsables de nuestros dolores que tenemos?

Bueno, igual no sabemos si sí es acuerdo o no, entonces pasamos a la siguiente propuesta:

Les proponemos el acuerdo de volver a reunirnos en un segundo encuentro el próximo año, pero no nada más aquí en tierras zapatistas, sino que también en sus mundos de cada quien, de acuerdo a sus tiempos y modos.

O sea que cada quien organice encuentros de mujeres que luchan o como le quieran llamar.

¿Están de acuerdo?

Bueno, no sabemos todavía qué respondieron, pero como quiera aquí serán bienvenidas, hermanas y compañeras.

Pero sí les pedimos que avisen con tiempo porque está cabrón que nos dicen que vienen quinientas y es que se les perdió un cero en el camino porque llegan cinco mil o más.

$Y$ viera que cuando vienen pueden decir que en sus mundos se reunieron, discutieron y acordaron lo que sea que acuerden.

O sea que lleguen más grandes en su corazón, en su pensamiento y en su lucha. 
Pero como quiera siempre serán bienvenidas, mujeres que luchan.

Gracias por escucharnos.

Ahora vamos a clausurar formalmente.

Tiene la palabra la comandanta Miriam:

Buenas noches, compañeras y hermanas.

Gracias, compañeras, gracias, hermanas de los países del mundo y de México que hicieron el esfuerzo de llegar hasta aquí en un rinconcito del mundo.

Es así como hemos terminado nuestro primer encuentro internacional político, artístico, deportivo y cultural de mujeres que luchan.

Siendo a las 20:36 hrs, hora zapatista, declaro clausurado nuestro primer encuentro.

Cuídense y que tengan buen viaje.

Desde Caracol 4 Torbellino de nuestras palabras, Morelia, Chiapas, México. Marzo 10 de 2018.

La metáfora de la vela, de la luz que nos dieron a cuidar, caló hondo en el contexto mexicano actual. "Acordamos vivir" fue la consigna, en un país con uno de los más altos índices de feminicidio, y con mayor índice de desapariciones. En marzo del 2019 el contexto del zapatismo había cambiado, la renuencia del mismo a formar parte del recambio político nacional, incluso su apreciación de que con el nuevo régimen la guerra contra los pueblos indígenas sería mayor, repercutió en que las compañeras enviaran una carta explicando porqué no convocarían a un segundo encuentro ni asistirían a ninguno.

Y en ese diálogo abierto surgió en 22 localidades distintas del país la iniciativa de hacer encuentros de mujeres que luchan. Desde la ciudad de México, y bajo el formato de dos días sólo mujeres en un campamento con foros, talleres y "actividades de movimiento", se replicó el sentido de encontrarse, mezclarse, hacer de la palabra revuelta una revuelta; ir reconociéndonos en una lucha común, desde las diversas resistencias y prácticas propositivas de transformación y sanación. Recuperar nuestra politicidad e imaginación para lo que sigue.

\section{Bibliografía}

Arruzza, Cinzia, Tithi Bhattacharya y Nancy Fraser, (2019): Manifiesto de un feminismo para el 99\%. Barcelona: Editorial Herder.

De Sousa Santos, Boaventura, (2010): Para descolonizar Occidente : más alla del pensamiento abismal. Buenos Aires : Consejo Latinoamericano de Ciencias Sociales CLACSO; Prometeo Libros. 
Revista Punto Género № 11. Junio de 2019 ISSN 0719-0417 / 18 - 33

Echeverría, Bolívar (2011): "Cuestionario sobre lo político", conversación con Luis Corral y José Ron (1980), en Bolívar Echeverría, ensayos políticos. Quito: Ministerio de Coordinación de la Política y Gobiernos Autónomos Descentralizados, p. 71-89.

González Casanova, Pablo (1965): La democracia en México. México: Editorial Era.

Millán, Márgara, Coordinadora (2014): Más allá del feminismo. Caminos para andar. México: Red de Feminismos Descoloniales.

Millán, Márgara (2014): Des-ordenando el género/ ¿Des-centrando la nación? El zapatismo de las mujeres indígenas y sus consecuencias. México: Ed. Del Lirio-UNAM, p. $126-132$.

Millán, Márgara, (2009): Revistas y políticas de traducción del feminismo mexicano contemporáneo. Estudos Feministas, p. 819-846.

Mohanty, Chandra,T. (2008): "Bajo los ojos de Occidente: academia feminista y discursos coloniales" en Liliana Suárez y Rosalva Aída hernández, eds. Descolonizando el feminismo. Teorías y prácticas desde los márgenes. Madrid: Ed. Cátedra, p. 117-163

Rivera Cusicanqui, Silvia (2010): Chïxinakax Utxiwa. Una reflexión sobre prácticas y discursos descolonizadores. Buenos Aires: Tinta Limón

Rivera Cusicanqui, Silvia, (2018): Un mundo ch'ixi es posible. Ensayos desde un presente en crisis. Buenos Aires: Ed. Tinta Limón.

Segato, Rita (2007): La Nación y sus Otros. Raza, etnicidad y diversidad religiosa en tiempos de Políticas de la Identidad. Buenos Aires: Ed. Prometeo.

Rita Segato, Rita, (2018): Manifiesto en cuatro temas, Critical Times, Vol 1, N. 1, Berkeley, Internacional Consortium of Critical Theory, p. 212 a 225.

Svampa, Maristella, (2018): Conferencia LNF: Ecofeminismos y feminismos populares, por Maristella Svampa - Canal Encuentro https://www.youtube.com/watch?v=TveVMH3Y4YI

Thomas, Sinclair. (2010): Prólogo a Silvia Rivera Cusicanqui, Violencias (re) encubiertas en Bolivia. La Paz: Ed. La mirada salvaje.

Varias Autoras, "Documento elaborado por las asesoras e invitadas del EZLN". (1997): Sara Lovera y Nelly Palomo, Las Alzadas. México: CIMAC, p. 301-324 